\title{
IFN- $\kappa$, a novel type I IFN, is undetectable in HPV-positive human cervical keratinocytes
}

\author{
Correne A DeCarlo ${ }^{1,7}$, Alberto Severini ${ }^{2}$, Lutz Edler ${ }^{3}$, Nicholas G Escott ${ }^{4}$, Paul F Lambert ${ }^{5}$, Marina Ulanova ${ }^{6}$ and \\ Ingeborg Zehbe ${ }^{1}$
}

Interferons (IFNs) are expressed by many cell types and play a pivotal role in the generation of immune responses against viral infections. IFN- $\kappa$, a novel type I IFN, displays a tight tropism for keratinocytes and specific lymphoid populations and exhibits functional similarities with other type I IFNs. The human papillomavirus (HPV), the etiological agent for cervical cancer, infects keratinocytes of the uterine cervix and has been shown to directly inhibit the IFN pathway. We evaluated IFN- $\kappa,-\beta$, and $-\gamma$ gene expression in HPV-negative normal and HPV-positive pre-malignant and malignant ex vivo cervical tissue covering the entire spectrum of cervical disease. Quantitative real-time polymerase chain reaction and methods previously optimized for detecting low-expressing genes in cervical tissue were used. In contrast to IFN- $\beta$ and $-\gamma$, IFN- $\kappa$ mRNA prevalence and levels were unexpectedly higher in diseased compared with normal whole cervical tissue with highest levels observed in invasive carcinoma tissue. Strikingly, laser capture microdissection revealed an absence of IFN- $\kappa$ mRNA in diseased epithelium, whereas stromal IFN- $\kappa$ was found exclusively in diseased tissue. IFN- $\gamma$ and IFN- $\beta$ were likewise found to be upregulated in diseased cervical stroma. Immunofluorescence supports the involvement of monocytes and dendritic cells in the stromal induction of IFNs in diseased tissue. Further, using three-dimensional raft cultures in which the viral life cycle can be mimicked, human keratinocytes transfected with full-length HPV16 displayed a significant decrease in IFN- $\kappa$ mRNA compared with non-transfected human keratinocytes. Altogether, these findings show that IFN- $\kappa$ is down-regulated in cervical keratinocytes harboring HPV, which may be a contributing factor in the progression of a cervical lesion.

Laboratory Investigation (2010) 90, 1482-1491; doi:10.1038/labinvest.2010.95; published online 17 May 2010

KEYWORDS: cervical tissue; human papillomavirus; interferon- $\kappa$; laser capture microdissection; quantitative real-time polymerase chain reaction

Interferons (IFNs) have a critical role in the generation of an innate immune response against invading pathogens and, as a consequence, are an approved therapy for many diseases ${ }^{1,2}$ including human papillomavirus (HPV)-associated diseases. $^{3,4}$ On infection, IFNs are synthesized and secreted to evoke anti-viral, ${ }^{5}$ anti-tumor, ${ }^{6}$ and immunoregulatory (reviewed in Stetson and Medzhitov ${ }^{7}$ ) activity conferring protection to neighboring cells. Two main classes of IFNs exist. The type I IFNs, consisting of IFN- $\alpha,-\beta,-\kappa,-\delta,-\varepsilon,-\omega$, and $-\tau$, share a common receptor and downstream signaling pathways (reviewed in Pestka et al. ${ }^{8}$ ) and are chiefly involved in generating anti-viral activity in response to pathogens. The sole member of the type II IFN subclass, IFN- $\gamma$, uses a discrete type II IFN receptor, ${ }^{9}$ distinct signaling pathways and is primarily involved in the regulation of immune and inflammatory responses. IFN- $\kappa$, a newly identified type I IFN, has been shown to signal through the type I IFN pathway ${ }^{10}$ and displays a tight tropism for keratinocytes, monocytes (MCs), and dendritic cells (DCs). ${ }^{10}$ IFN- $\kappa$ has been shown to display similar anti-viral activity to other type I IFNs. ${ }^{11}$ However, it is distinct from other type I IFNs, as it seems to signal in a discrete autocrine, rather than paracrine, cell-associated manner. ${ }^{11}$ Due to its distinctive nature, IFN- $\kappa$ could offer improvements over current IFN treatment regimes

\footnotetext{
${ }^{1}$ Thunder Bay Regional Research Institute, Probe Development and Biomarker Exploration, Thunder Bay, ON, Canada; ${ }^{2}$ National Microbiology Laboratory, Public Health Agency of Canada, Winnipeg, MB, Canada; ${ }^{3}$ Department of Biostatistics, Deutsches Krebsforschungszentrum, Heidelberg, Germany; ${ }^{4}$ Thunder Bay Regional Health Sciences Centre, Thunder Bay, ON, Canada; ${ }^{5}$ McArdle Laboratory for Cancer Research, University of Wisconsin, Madison, WI, USA and ${ }^{6}$ Northern Ontario School of Medicine, Medical Sciences Division, Thunder Bay, ON, Canada

Correspondence: Dr I Zehbe, PhD, DSc, Thunder Bay Regional Research Institute, Probe Development and Biomarker Exploration, 980 Oliver Road, Thunder Bay, ON P7B 6V4, Canada.

E-mail: zehbei@tbh.net

${ }^{7}$ Current address: Department of Psychology, University of Victoria, Victoria, BC Canada.

Received 30 September 2009; revised 19 March 2010; accepted 24 March 2010
} 
where substantial side-effects may limit the therapeutic potential of IFNs. ${ }^{12}$ Earlier studies using ex vivo human skin biopsies indicate that IFN- $\kappa$ expression is completely absent in normal skin but strongly induced in allergic contact dermatitis and planus-affected skin. ${ }^{13}$

HPV is a double-stranded DNA virus, which infects the squamous epithelium of the uterine cervix. Most infections are effectively cleared by the immune system; however, a proportion of women are unable to clear the infection resulting in cervical dysplasia and, in some cases, cervical cancer. HPV has the ability to directly interact with and inhibit the function of many molecules involved in the IFN pathway, which may, in part, explain why HPV infections can be sustained in the host without initiating an effective immune response. HPV interacts with molecules involved in IFN gene regulation, such as the IFN regulatory factors ${ }^{14,15}$ and downstream IFN-elicited pathways, such as $\mathrm{p} 48,{ }^{16}$ and the Janus kinase/signal transducers and activators of transcription. ${ }^{17,18}$ Accordingly, it has been shown that type I IFNs are downregulated in keratinocytes infected with HPV $16^{19}$ and in HPV + cervical carcinoma cell lines, ${ }^{20}$ and IFN- $\gamma$ expression is significantly decreased in cervical carcinoma tissue. $^{21,22}$ Convincing evidence exists illustrating the inhibitory effects of HPV on IFNs. However, no investigations into the effect of a DNA virus, such as HPV, on IFN- $\kappa$ expression exist for the full spectrum of cervical disease.

After a viral infection, the host uses a variety of cellular sensors, such as toll-like receptors, composed of membrane spanning proteins with domains for the detection of pathogen-associated molecular patterns. The complex signaling pathways elicited stimulate nuclear factor of $\kappa \mathrm{B}$ and ultimately result in the transcriptional activation and production of IFNs. The analysis of IFN transcript levels in ex vivo cervical tissue, therefore, likely represents a direct examination of the downstream effects of HPV infection on IFN regulation and thus is the rationale for our focus on the IFN transcriptome, rather than the proteome, in the current study.

Cervical tissue, the main constituent of which is the squamous keratinocyte, represents an ideal context in which to study IFN- $\kappa$ expression in response to a viral infection as HPV infects keratinocytes, the cell type that chiefly expresses IFN $-\kappa$. Earlier research into the effects of HPV16 on the IFN transcriptome revealed a detrimental effect of the virus on these genes, ${ }^{19}$ an effect we are endeavoring to validate for IFN- $\beta$ and IFN- $\gamma$, and notably for novel IFN- $\kappa$, in ex vivo cervical tissue. Given this knowledge, we hypothesize that IFN- $\kappa$ will likewise be inhibited by the virus; results that will be confirmed through the isolation of cervical keratinocytes. Although earlier studies have investigated IFN- $\kappa$ in skin keratinocytes and cell lines, to our knowledge, IFN- $\kappa$ expression has never been investigated in isolated cervical epithelium and stroma using tissue microdissection.

Here we report, using the full spectrum of cervical carcinogenesis, a comprehensive analysis of the effects of HPV infection on IFN- $\kappa$ gene expression in relation to other IFNs in normal, dysplastic, and carcinoma ex vivo whole cervical biopsy tissue and microdissected cervical epithelium and stroma. IFN- $\kappa$ gene expression levels and prevalence were significantly higher in diseased $v s$ healthy HPV-negative whole biopsy cervical tissue. Laser capture microdissection (LCM) revealed the involvement of the cervical stroma, and not the keratinocytes, in the HPV-induced expression of IFN- $\kappa$ as well as the absence of IFN- $\kappa$ in diseased epithelium. Immunofluorescence implicates MCs and DCs in the induction of IFN $-\kappa$ expression in diseased stroma. These findings show that ex vivo cervical keratinocytes harboring HPV are IFN- $\kappa$ deficient, which could contribute to the progression of a cervical lesion.

\section{MATERIALS AND METHODS Sample Preparation}

Cervical biopsies were obtained with written consent from women attending the Colposcopy Clinic at the Thunder Bay Regional Health Sciences Centre (TBRHSC) between November 2005 and November 2006. The study has been approved by the local Research Ethics Team at the TBRHSC (\#21.05). Biopsies were snap frozen in liquid nitrogen and immediately transferred to $-80^{\circ} \mathrm{C}$. Tissue was sectioned on a cryostat (Leica CM1850, Leica Microsystems, Richmond Hill, ON, Canada), maintaining tissue temperature at $-20^{\circ} \mathrm{C}$ using Tissue Tek embedding medium (O.C.T. Compound, Sakura Finetek, Torrance, CA, USA) and sectioned to $10 \mu \mathrm{m}$ thickness. For full-biopsy analysis, 10 sections from each specimen were placed in a $1.5-\mathrm{ml}$ microcentrifuge tube for subsequent RNA extraction. For LCM analysis, $8 \mu \mathrm{m}$-thick tissue sections were cut, adhered to uncharged slides, and kept in $-20^{\circ}$ before LCM preparation. All tweezers, brushes, and surfaces were cleaned with DEPC-treated $70 \%(\mathrm{v} / \mathrm{v})$ ethanol between specimens to reduce RNAse activity and RNA cross-contamination.

All 110 samples collected in this study were thoroughly tested for RNA integrity. Inclusion of a sample was based on: suitable RNA integrity, as measured on the Experion Automated Electrophoresis System (Bio-Rad, Mississauga, ON, Canada), for both whole biopsy and LCM material separately, availability of sufficient amounts of epithelium and stroma in the section to allow for tissue microdissection and an unbiased analysis, the amount of sample RNA available for use in quantitative real-time polymerase chain reaction (qRTPCR), and a morphological diagnosis that corresponds to the HPV infection status. From 110 cervical biopsy specimens collected, 38 corresponded to our stringent tissue requirements and were therefore suitable for use in this study. Samples were used either for whole tissue analysis, LCM analysis or both, depending on the availability of tissue. In total, six cervical carcinoma specimens were processed, with only four being suitable for LCM analysis. Initially, four carcinoma whole biopsy specimens, with equal parts epithelium and stroma, were analyzed. Two of these samples 
Table 1 Sample size

\begin{tabular}{|c|c|c|c|c|c|}
\hline$\#$ & $\begin{array}{l}\text { Case } \\
\text { ID }\end{array}$ & $\begin{array}{l}\text { Morphological } \\
\text { diagnosis }\end{array}$ & $\begin{array}{l}\text { Whole } \\
\text { biopsy }\end{array}$ & LCM & $\begin{array}{l}\text { HPV infection } \\
\text { status }\end{array}$ \\
\hline 1 & $\mathrm{~N} 1$ & Normal & $\sqrt{ }$ & $\sqrt{ }$ & Negative \\
\hline 2 & N2 & Normal & $\sqrt{ }$ & - & Negative \\
\hline 3 & N3 & Normal & $\sqrt{ }$ & $\sqrt{ }$ & Negative \\
\hline 4 & N4 & Normal & $\sqrt{ }$ & $\sqrt{ }$ & Negative \\
\hline 5 & N5 & Normal & $\sqrt{ }$ & $\sqrt{ }$ & Negative \\
\hline 6 & N6 & Normal & $\sqrt{ }$ & $\sqrt{ }$ & Negative \\
\hline 7 & N7 & Normal & - & $\sqrt{ }$ & Negative \\
\hline 8 & N8 & Normal & - & $\sqrt{ }$ & Negative \\
\hline 9 & N9 & Normal & - & $\sqrt{ }$ & Negative \\
\hline 10 & N10 & Normal & - & $\sqrt{ }$ & Negative \\
\hline 11 & N11 & Normal & - & $\sqrt{ }$ & Negative \\
\hline 12 & $\mathrm{~N} 12$ & Normal & - & $\sqrt{ }$ & Negative \\
\hline 13 & D1 & LSIL & $\sqrt{ }$ & - & $\begin{array}{l}\text { HR HPV+ } \\
\text { Type not } \\
\text { determined }\end{array}$ \\
\hline 14 & D2 & LSIL & $\sqrt{ }$ & $\sqrt{ }$ & Type 31 \\
\hline 15 & D3 & LSIL & $\sqrt{ }$ & - & Type 39 \\
\hline 16 & D4 & LSIL & $\sqrt{ }$ & - & Type 16 \\
\hline 17 & D5 & LSIL & $\sqrt{ }$ & - & Type 40 \\
\hline 18 & D6 & LSIL & $\sqrt{ }$ & - & Types 6, 51 \\
\hline 19 & D7 & LSIL & $\sqrt{ }$ & $\sqrt{ }$ & Type 51 \\
\hline 20 & D8 & LSIL & $\sqrt{ }$ & $\sqrt{ }$ & Types 66, 82 \\
\hline 21 & D9 & LSIL & - & $\sqrt{ }$ & Types 6, 66 \\
\hline 22 & D10 & HSIL & $\sqrt{ }$ & - & Types 26, 31 \\
\hline 23 & D11 & HSIL & $\sqrt{ }$ & $\sqrt{ }$ & Type 16 \\
\hline 24 & D12 & HSIL & $\sqrt{ }$ & - & $\begin{array}{l}\text { HR HPV+ } \\
\text { Type not } \\
\text { determined }\end{array}$ \\
\hline 25 & D13 & HSIL & $\sqrt{ }$ & - & Type 52 \\
\hline 26 & D14 & HSIL & $\sqrt{ }$ & - & Types $18,33,45$ \\
\hline 27 & D15 & HSIL & $\sqrt{ }$ & $\sqrt{ }$ & Type 16 \\
\hline 28 & D16 & HSIL & $\sqrt{ }$ & - & Types 16, 39 \\
\hline 29 & D17 & HSIL & $\sqrt{ }$ & $\sqrt{ }$ & Type 33 \\
\hline 30 & D18 & HSIL & - & $\sqrt{ }$ & Type 16 \\
\hline 31 & D19 & HSIL & - & $\sqrt{ }$ & Type 16 \\
\hline 32 & $\mathrm{D} 20$ & HSIL & - & $\sqrt{ }$ & Type 39 \\
\hline 33 & $\mathrm{C} 1$ & Carcinoma & $\sqrt{ }$ & - & Type 16 \\
\hline 34 & $\mathrm{C} 2$ & Carcinoma & $\sqrt{ }$ & $\sqrt{ }$ & Type 16 \\
\hline 35 & $\mathrm{C} 3$ & Carcinoma & $\sqrt{ }$ & - & Type 18 \\
\hline 36 & $\mathrm{C} 4$ & Carcinoma & $\sqrt{ }$ & $\sqrt{ }$ & $\begin{array}{l}\text { HR HPV+ } \\
\text { Type not }\end{array}$ \\
\hline
\end{tabular}

Table 1 Continued

\begin{tabular}{llllll}
\hline \# & $\begin{array}{l}\text { Case } \\
\text { ID }\end{array}$ & $\begin{array}{l}\text { Morphological } \\
\text { diagnosis }\end{array}$ & $\begin{array}{l}\text { Whole } \\
\text { biopsy }\end{array}$ & LCM & $\begin{array}{l}\text { HPV infection } \\
\text { status }\end{array}$ \\
\hline 37 & C5 & Carcinoma & - & $\sqrt{ }$ & $\begin{array}{l}\text { HR HPV+ } \\
\text { Type not } \\
\text { determined }\end{array}$ \\
& C6 & Carcinoma & - & $\sqrt{ }$ & $\begin{array}{l}\text { HR HPV+ } \\
\text { Type not } \\
\end{array}$ \\
& & & & determined \\
& & & & & \\
\end{tabular}

Thirty-eight FR cervical biopsies were categorized into three groups: normal tissue, case IDs N1-N12; dysplastic tissue (LSIL and HSIL), case IDs D1-D20; and invasive carcinoma tissue, case IDs C1-C6. The use of the specimen in whole biopsy or LCM analysis is shown along with the HPV infection status. LSIL denotes low-grade squamous intraepithelial lesion and HSIL denotes highgrade squamous intraepithelial lesion.

with remaining tissue were subsequently processed for LCM. Tissue architecture in the remaining two samples precluded clean isolation of epithelium from dermal stroma using LCM due to infiltrating tumor cells in the stroma. To further validate epithelial findings, two additional carcinoma specimens consisting predominately of tumor cells were used for LCM.

The 38 samples used in this study along with their HPV infection status are listed in Table 1. In total, 12 of 38 samples were negative for HPV and diagnosed as morphologically normal (Table 1). Of the 26 diseased samples, 9 were diagnosed as low-grade lesions (LSIL), 11 were high-grade lesions (HSIL), and 6 were invasive carcinomas. Importantly, all normal tissue was negative for HPV infection and all diseased tissue was positive for HPV infection. Of the 26 diseased samples, 25 were infected with a high-risk (HR) HPV type and 1 sample was infected with a low-risk (LR) HPV type, as shown in Table 1.

\section{HPV Typing and Specimen Classification}

Ten tissue sections were cut, immediately after sections were taken for RNA extraction, and placed into $1.5 \mathrm{ml}$ microcentrifuge tubes for DNA extraction. DNA extraction was performed using the Qiagen QIAamp DNA micro kit (Qiagen, Mississauga, ON, Canada) following the manufacturer's protocol for tissue samples. Samples were HPV genotyped using Luminex hybridization at the National Microbiology Laboratory (Winnipeg, Manitoba, Canada). Briefly, the method amplifies DNA from mucosal HPV types by a nested PCR method using PGMY primers ${ }^{23}$ followed by GP5 + /GP6 + amplification. The products were labeled with the fluorophore phycoerythrin and hybridized to sortable microspheres, carrying specific probes for 45 mucosal HPV types. The probes are sensitive and specific for each type without cross-hybridization. ${ }^{24,25}$ In addition, PCR analysis ${ }^{26}$ and p16-based testing using the CINtec p16 immunocytochemistry staining kit (CINtec p16INK4a Cytology 
Kit; mtm laboratories, Westborough, MA, USA) were performed at the regional Cancer Care Laboratory of the TBRHSC. For histopathological diagnosis, sections were cut and processed for hematoxylin and eosin staining ${ }^{27}$ and diagnosed by the same pathologist (NE). Tissue samples were diagnosed as morphologically normal, low/high-grade lesion and cervical carcinoma tissue. For this study, samples were categorized as; HPV negative and morphologically normal (normal; $n=12$ ), HPV positive and morphologically dysplastic (dysplasia; $n=20$ ) and HPV positive and morphologically malignant (carcinoma; $n=6$ ).

\section{Human Keratinocytes and Organotypic Raft Cultures}

Primary human mucosal keratinocytes (PHMK) were obtained from ScienCell (Carlsbad, CA, USA) and cultured according to the recommendations by the supplier. NIKS, derived from normal, spontaneously immortalized human keratinocyte $^{28}$ were cultured as described earlier. ${ }^{29}$ For dsRNA stimulation, $20 \mu \mathrm{g} / \mathrm{ml}$ Poly (I:C)-LMW (InvivoGen, San Diego, CA, USA) was incubated with keratinocytes for 0, $8,16,24$, and $48 \mathrm{~h}$. For growing three-dimensional organotypic raft cultures, NIKS were grown on artificial stroma with collagen and fibroblasts ${ }^{30}$ in triplicate, as described. ${ }^{29}$ Fibroblast containing collagen rafts without NIKS served as negative controls. ${ }^{29}$ After 14 days in the liquid/air interface, cultures were harvested. For qPCR and immunohistochemistry, cultures were snap frozen in liquid nitrogen or fixed in $4 \%$ buffered formaldehyde solution, respectively. For total RNA extraction, rafts were pulverized with liquid nitrogen and pestles and thereafter extracted as recently described. ${ }^{31}$ Proof of the viral life cycle during the growth of the rafts was obtained using a monoclonal antibody against a crucial capsid protein, L2 (a kind gift from Martin Mueller, Deutsches Krebsforschungszentrum, Heidelberg, Germany), which is only expressed during the viral life cycle.

\section{RNA Extraction and Integrity Assessment}

RNA was extracted from $10 \times 10 \mu \mathrm{m}$ thick tissue sections, microdissected cervical cells or keratinocytes as described earlier. ${ }^{31}$ The quality and quantity of RNA extracted from cervical tissue was assessed using the Bio-Rad Experion Automated Electrophoresis System. RNA integrity from samples was assessed as described earlier. ${ }^{31}$

\section{Laser Capture Microdissection}

Tissue specimens were prepared for LCM using the Arcturus Histogene Frozen Section Staining Kit, as described earlier. ${ }^{31}$ Up to 5000 captures ( $\sim 25000$ cells) of both epithelium and stroma for each tissue specimen (where possible) were taken. Macro caps were cleaned of unwanted debris using CapSure pads (Arcturus) before being deposited into $0.5 \mathrm{ml}$ microfuge tubes for RNA extraction. The laser spot size was consistently $15 \mu \mathrm{m}$ in diameter, whereas the laser power and duration ranged from 80 to $95 \mathrm{~mW}$ and $0.65-0.8 \mathrm{~ms}$ duration between specimens. Images of the excision process have been reported earlier. $^{31}$

\section{Reverse Transcription and Transcript Amplification}

RNA isolated from samples was reverse transcribed to complementary DNA (cDNA) using the High Capacity cDNA Archive Kit (Applied Biosystems) according to the manufacturer's directions with random hexamer primers. Seventy-two nanograms of RNA from whole tissue specimens and of the keratinocytes was reverse transcribed at $4 \mathrm{ng} / \mu \mathrm{l}$, whereas RNA isolated from microdissected samples was reverse transcribed at a minimum of $0.6 \mathrm{ng} / \mu \mathrm{l}$ (ranging from 0.6 to $2.8 \mathrm{ng} / \mu \mathrm{l}$ ), depending on the acquired LCM sample RNA concentration.

Twelve and a half microlitres of cDNA from all reactions (corresponds to $50 \mathrm{ng}$ for whole biopsy sections and keratinocytes and between 7.5 and $35 \mathrm{ng}$ from 5000 LCM captures) was amplified using the TaqMan PreAmp Master Mix Kit (Applied Biosystems). Gene amplification uniformity was assessed as described earlier. ${ }^{31}$

\section{Quantitative Real-Time Polymerase Chain Reaction}

Reactions were carried out according to the qRT-PCR protocol specified in the TaqMan PreAmp Master Mix Kit. Triplicate reactions of $25 \mu \mathrm{l}$ volume were added to a 96optical well plate (Applied Biosystems) and incubated at standard qRT-PCR conditions $\left(50^{\circ} \mathrm{C}\right.$ for $5 \mathrm{~min}, 95^{\circ} \mathrm{C}$ for $10 \mathrm{~min}$ and then cycled at $95^{\circ} \mathrm{C}$ for $15 \mathrm{~s}$ and $60^{\circ} \mathrm{C}$ for $1 \mathrm{~min}$ for 40 cycles (detection limit)). qRT-PCR was repeated for $10 \%$ of ex vivo human samples to ensure result reliability. In vitro samples were run in triplicate with representative results shown. Target genes were normalized to the housekeeping gene hypoxanthine phosphoribosyltransferase1 (HPRT1), as earlier results indicate its expression is unaffected by HPV infection. ${ }^{31}$ TaqMan gene expression on demand assays for HPRT1, HPV E6, HPV E7, IFN- $\gamma$, IFN- $\beta$, and IFN- $\kappa$ were used (Applied Biosystems). Negative controls where cDNA was omitted or the enzyme was missing in the RT reaction were run to monitor for contamination or non-specific primer binding. A positive control was included on every plate to control for variation between runs. Relative quantification of target genes was performed using auto Ct and baseline settings and a threshold of 0.20 (Applied Biosystems 7300/7500/7500 Fast Real-Time PCR System Software). Values are presented as 'relative' values as all $\mathrm{Ct}$ values are normalized to HPRT-1 and their inverse values are compared.

\section{Immunofluorescence}

Antigen detection was performed with primary mouse monoclonal antibodies against CD11c and CD14 (clones BU15 and 61D3, Abcam, Cambridge, MA, USA) as well as L2 to monitor for DCs, MCs, and the HPV L2 capsid protein, respectively. All antibodies were diluted 1:100 in Chemmate antibody diluents (DAKO, Hamburg, Germany), added to the 
tissue sections and incubated at $4^{\circ} \mathrm{C}$ over night. Tissue was then washed three times in cold $1 \times$ PBS before the addition of the secondary antibody. Alexa Fluor 594 (against mouse) antibodies raised in donkey (Molecular Probe, Invitrogen, Burlington, ON, Canada) and diluted 1:800 in Chemmate were added to the tissue and incubated in the dark at room temperature for $1 \mathrm{~h}$. Tissue was washed three times in cold $1 \times$ PBS and twice in distilled water before mounting in VectaShield mounting medium with DAPI (Molecular Probes). Fluorescence imaging was performed using a Nikon fluorescence microscope (Nikon Eclipse 80i with digital imaging head) and a cooled color CCD camera (Nikon DXM1200C). Channels for detecting $594 \mathrm{~nm}$ (where positive signals would be expected) and $488 \mathrm{~nm}$ (where no signal would be expected) fluorescence were used to distinguish specific (positive) signals from autofluorescence. Images were created using an original $\times 200$ magnification.

\section{Statistical Analysis}

Prevalence of IFN gene expression was described using proportions observed in the three tissue models, namely normal $(n=12)$, dysplastic (LSIL + HSIL, $n=20)$, and cervical carcinoma $(n=6)$. Differences in IFN gene expression prevalence between normal, dysplasia, and carcinoma tissue was assessed using the Cochrane-Armitage trend test ${ }^{32}$ when analyzing the three types together and using Fisher's exact test for pairwise comparison of two types. Gene expression levels were described using means and s.d. Differences in gene expression levels were assessed using the JonckheereTerpstra $^{33}$ test for trends when analyzing the three types together and using the Student's $t$-test for pairwise comparison of two types and keratinocytes. $P$-values for all statistical comparisons are reported with at most two significant digits.
Statistical significance is stated when $P<0.05$. All tests are two-sided if not otherwise indicated and based on the 'exact' version. Calculations were performed using Microsoft Excel version 12 (2008) and Cytel Studio StatXact7 version 7.0.0 (2005), Cytel Software Corporation (http://www.cytel.com) and the SAS statistical analysis package (Version 9.1.2, http:// support.sas.com) for statistical tests.

\section{RESULTS}

\section{Unlike IFN- $\beta$ and IFN- $\gamma$, IFN- $\kappa$ Prevalence and Gene Expression is Increased in Diseased vs Healthy Full-Biopsy Cervical Tissue}

Earlier studies indicate that type I and II IFNs are negatively influenced by HPV infection. ${ }^{19,21}$ Accordingly, we theorized that the expression of IFN- $\kappa$, a type I IFN with a strong tropism for keratinocytes, would likewise be reduced on HPV infection ex vivo. In initial experiments, we used whole ex vivo human cervical biopsy specimens $(n=26)$ to exactly model the in vivo microenvironment within the uterine cervix using QRT-PCR and methods optimized for IFN- $\kappa$ detection. ${ }^{31}$ Prevalence was determined by the presence or absence of a detectable Ct (threshold cycle) value using qRT-PCR. In contrast to the downregulation of IFN- $\kappa$ found in HPV-immortalized keratinocytes, ${ }^{34}$ IFN- $\kappa$ mRNA prevalence and gene expression level was significantly increased in HPV-infected diseased full-biopsy cervical tissue. A 4.2fold increase in IFN- $\kappa$ mRNA prevalence between normal (1/6) and diseased cervical tissue (14/20) was found. IFN- $\kappa$ gene expression in whole cervical biopsy specimens was detected significantly more often in diseased tissue (dysplastic tissue $10 / 16 ; 63 \%$ and carcinoma tissue $4 / 4 ; 100 \%$ ) than normal tissue $(1 / 6 ; 16.7 \%)(P<0.033$ one-sided Fisher's exact test), as shown numerically in Table 2 . On a trend analysis, the prevalence of IFN $\kappa$ was found to be statistically

Table 2 IFN expression in cervical epithelium and stroma

Relative expression of IFN genes in LCM material

\begin{tabular}{|c|c|c|c|c|}
\hline Gene & Normal & Dysplasia & Carcinoma & Trend \\
\hline \multicolumn{5}{|c|}{ Cervical epithelium } \\
\hline IFN- $\kappa$ & $0.03 \pm 0.04$ & - & - & Decreased expression with disease \\
\hline IFN- $\beta$ & $0.09 \pm 0.03$ & $0.07 \pm 0.05$ & $0.10 \pm 0.02$ & No significant $\Delta$ with disease \\
\hline $\mathrm{IFN}-\gamma$ & $0.11 \pm 0.04$ & $0.12 \pm 0.06$ & $0.13 \pm 0.04$ & No significant $\Delta$ with disease \\
\hline
\end{tabular}

Cervical stroma

$\begin{array}{lllll}\text { IFN- } \kappa & - & 0.01 \pm 0.03 & 0.08 \pm 0.01 & 0.14 \pm 0.01 \\ \text { IFN- } \beta & 0.10 \pm 0.04 & 0.11 \pm 0.05 & 0.19 \pm 0.05 & 0.22 \pm 0.11\end{array}$

Average relative gene expression of IFN $-\kappa,-\beta$, and $-\gamma$ in normal, dysplastic, and carcinoma epithelium and stroma isolated using LCM and measured using qRT-PCR. No IFN- $\kappa$ expression was detected in diseased epithelium or in normal stroma. Numerical values represent the average 1 over $C t$ value \pm s.d. $\Delta$ denotes change in gene expression (not calculated). 


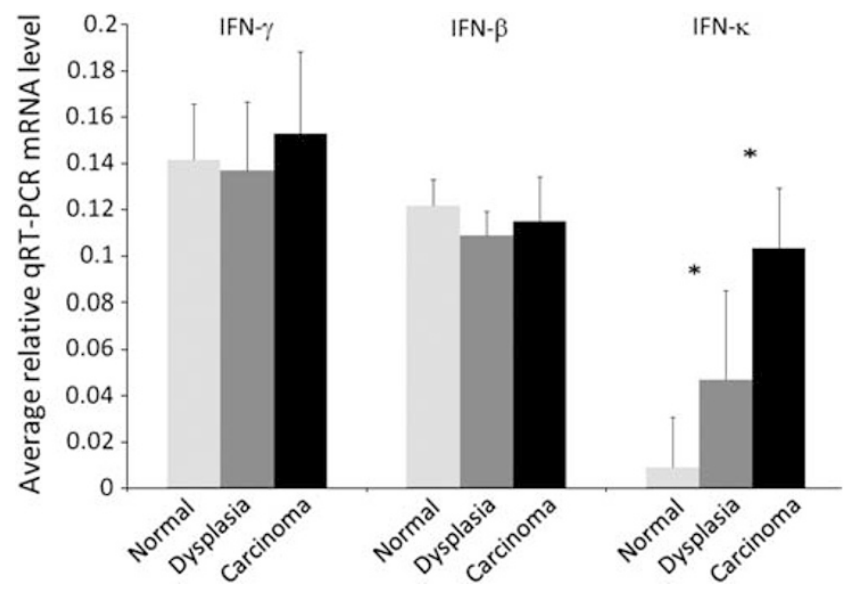

Figure 1 IFN expression in cervical whole biopsy specimens (ex vivo). Relative gene expression of IFN $-\gamma,-\beta$, and $-\kappa$ in normal, dysplastic, and carcinoma tissue measured using qRT-PCR. Stars indicate a statistically significant difference in expression levels between groups. Values are presented as averages of 1 over Ct values + s.d.

significantly increasing from normal to dysplastic to carcinoma tissue (Cochrane-Armitage test for trend: $P<0.016$ ). Moreover, the expression levels of IFN- $\kappa$ exhibited a highly statistically significant increasing trend from normal tissue through dysplastic lesions to carcinoma tissue (JonckheereTerpstra test for trend: $P=0.00076$, one-sided for increase). A zero Ct value is a qualitative finding (absence of expression) and to incorporate this meaningful finding into the results, zero values were included in the quantitative analysis. The mean relative expression levels were $\sim 6.0$-fold higher in diseased tissue compared with normal tissue $(0.06 \pm 0.04$ vs $0.01 \pm 0.02$ ), and more specifically 5.0-fold and 10.0-fold higher in dysplastic and carcinoma tissue compared with normal tissue $(0.05 \pm 0.04$ and $0.10 \pm 0.03$ vs $0.01 \pm 0.02)$, respectively, and 2.0-fold higher in carcinoma tissue compared with dysplastic tissue $(0.10 \pm 0.03$ vs $0.05 \pm 0.04)$ (Figure 1). No significant differences were found in IFN- $\kappa$ mRNA levels when samples were stratified by HPV type (LR vs HR; data not shown).

In contrast, IFN- $\beta$ and IFN- $\gamma$ prevalence and mRNA levels did not differ between normal and diseased tissue. IFN- $\beta$ was found in all $(26 / 26 ; 100 \%)$ samples analyzed, as seen in Table 2 . On a trend analysis, IFN- $\beta$ expression levels did not differ between normal $(0.12 \pm 0.01)$, dysplastic $(0.11 \pm 0.01)$, and carcinoma groups $(0.11 \pm 0.02)$ (Jonckheere-Terpstra test for trend: $P=92$, one-sided for increase, n.s.), indicating that IFN- $\beta$ transcription is not significantly altered with cervical disease progression, as shown in Figure 1. Like IFN- $\beta$, IFN- $\gamma$ was found in all $(26 / 26 ; 100 \%)$ samples analyzed, as seen in Table 2. Much intra-individual variability in IFN- $\gamma$ gene expression levels was evident within groups. However, a trend analysis did not reveal any differences in IFN- $\gamma$ gene expression levels between normal $(0.14 \pm 0.02)$, dysplastic $(0.14 \pm 0.03)$, and carcinoma groups $(0.15 \pm 0.04)$
(Jonckheere-Terpstra test for trend: $P=0.34$, n.s.), as shown in Figure 1.

The most striking result obtained from the whole biopsy analysis was the unpredicted increase in IFN- $\kappa$ gene expression and prevalence in diseased tissue; results that lead to further investigations to uncover the tissue subcompartment involved in the observed expression.

\section{IFN- $\boldsymbol{\kappa}$ is Absent in Diseased Cervical Keratinocytes and Present only in Diseased Stroma}

LCM was used to isolate keratinocytes from underlying dermal stroma in whole cervical biopsy specimens $(n=24)$ to reveal the cell type responsible for the observed IFN- $\kappa$ induction. Intuitively, changes in IFN- $\kappa$ expression with disease progression were expected to be confined to the cervical keratinocytes. Strikingly, in the epithelium, there was a significant decrease in IFN- $\kappa$ prevalence in diseased compared with normal tissue (Fisher's exact test: $P=0.031$, two-sided), as IFN- $\kappa$ was found to be exclusive to normal epithelium and completely absent in all dysplastic and carcinoma epithelial samples tested. On a trend analysis, the prevalence of IFN- $\kappa$ was found to significantly decrease from normal to dysplastic to carcinoma epithelium (CochraneArmitage test for trend: $P<0.031$, one-sided) with prevalent IFN- $\kappa$ mRNA levels found solely in normal epithelium. In the stroma, although the prevalence of IFN- $\kappa$ mRNA did not differ statistically between normal and diseased samples (Fisher's exact test, $P=0.21$, two-sided, (n.s.)), IFN- $\kappa$ mRNA was strikingly absent in all normal cases yet prevalent exclusively in diseased cases. In addition, it is well known that IFNs stimulate the production of downstream cytokines. To investigate the impact of the observed HPV-associated IFN induction, we analyzed the gene expression levels of pro- and anti-inflammatory cytokines IL-2, $-4,-6,-8$, and -10 , as well as tumor growth factor- $\beta$ and tumor necrosis factor- $\alpha$ in epithelial and stromal compartments of diseased and healthy cervical tissue. Interestingly, out of the seven cytokines measured in both tissue compartments, qRT-PCR analysis revealed a decreasing trend in IL-10 mRNA levels from normal to dysplastic to carcinoma epithelium (JonckheereTerpstra test for trend: $P=0.015$, one-sided for increase) as well as an increasing trend in IL-10 mRNA levels from normal to dysplastic to carcinoma stroma (JonckheereTerpstra test for trend: $P=0.044$, one-sided for increase).

As IFN- $\kappa$ was undetectable in dysplastic or malignant keratinocytes derived from ex vivo HPV-infected cervical tissue, we wanted to provide evidence that this is due to the inhibitory action by HPV. As a host defense mechanism during viral infection, the innate immune system is normally triggered by producing type I IFNs, ${ }^{35}$ including IFN $-\kappa .{ }^{10}$ However, HPV has been shown to interact and inhibit the IFN pathway. ${ }^{14-16}$ If our theory that the presence of HPV triggers IFN- $\kappa$ suppression in ex vivo cervical keratinocytes is correct, then related in vitro environments should generate similar results. To mimic the HPV infectious cycle and 

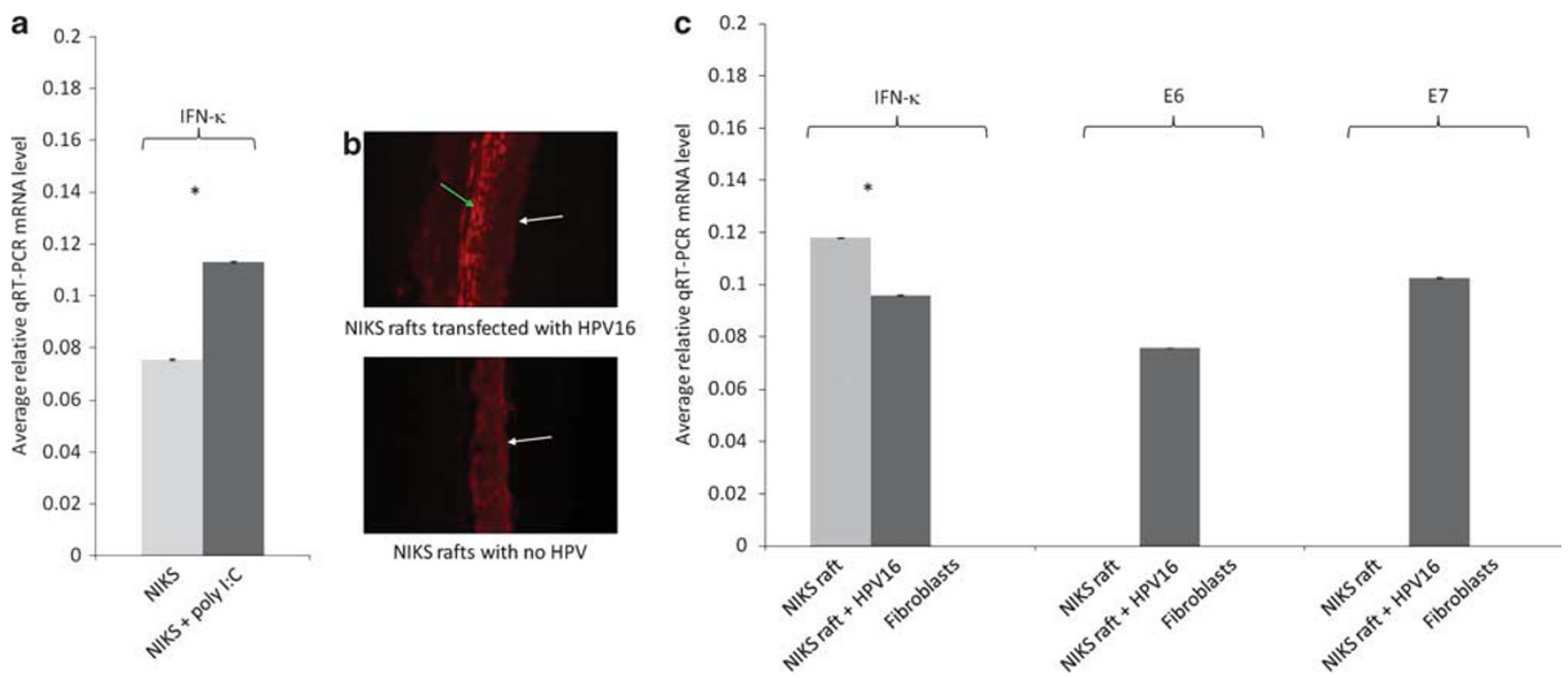

Figure 2 IFN- $\kappa$ expression in human keratinocytes (in vitro). (a) Relative IFN- $\kappa$ expression measured using qRT-PCR in normal NIKS and NIKS incubated with poly I:C for $24 \mathrm{~h}$. (b) HPV L2 capsid protein immunofluorescence in differentiated keratinocytes. White arrows indicate the basal cell layer, whereas the green arrow indicates the L2-positive cells in the upper part of the epithelium. (c) Relative expression of IFN- $\kappa$, E6, and E7 measured using qRT-PCR in keratinocytes from normal NIKS rafts, keratinocytes from NIKS rafts harboring full-length HPV16 and fibroblasts rafts (equivalent of tissue stroma). Representative experiments are shown. Stars indicate a statistically significant difference in expression levels between conditions. Values are presented as averages of 1 over Ct values + s.d.

because HPV particles can only be produced in stratified, differentiated epithelium, we used an earlier established model based on a spontaneously immortalized keratinocyte cell line derived from skin, NIKS, which has retained all features necessary for the propagation of this virus. ${ }^{30}$ Another reason why the NIKS cell line was chosen is because experiments may be repeated multiple times within the identical genetic background. This would be impossible using primary keratinocytes that senesce after $\sim 10$ passages. ${ }^{36}$ To ensure that IFN $-\kappa$ can be induced in NIKS and in PHMK, that is the cell type in which most cervical cancers arise, both keratinocyte cell types were treated with dsRNA using a time course from 0 to $48 \mathrm{~h}$. As expected, both NIKS and PHMK were able to induce IFN- $\kappa$ peaking after 8-24h. Incubation with dsRNA for $24 \mathrm{~h}$ induced IFN $-\kappa$ by threefold in PHMK $(0.196 \pm 0.0001$ vs $0.585 \pm 0.0002 ; P<0.001$, data not shown) and by 1.5 -fold in NIKS $(0.076 \pm 0.0003 v s$ $0.113 \pm 0.0003 ; P<0.001$; Figure $2 \mathrm{a})$. Next, we analyzed IFN- $\kappa$ mRNA levels in three-dimensional raft cultures with NIKS alone or NIKS transfected with full-length HPV16. Immunofluorescence using a monoclonal antibody against the HPV16 L2 capsid protein was performed to confirm the presence of an active viral life cycle. Positive signals, corresponding to the epithelial part where HPV viral particles are mostly produced, were found within the upper part of the epithelium (Figure $2 \mathrm{~b}$ ). IFN- $\kappa$ mRNA production was not only repressed, it was also reduced by 1.23 -fold in HPV16positive rafts $(0.118 \pm 0.0001$ vs $0.096 \pm 0.0002 ; P<0.001$; Figure $2 c)$. Thus, when mimicking an RNA virus infection in NIKS, IFN- $\kappa$ production is increased (1.5-fold), yet in the presence of HPV, IFN- $\kappa$ production is decreased (1.25-fold). As anticipated, both HPV viral genes, E6 and E7, were expressed in HPV16 + rafts (Figure 2c), supporting earlier findings that their presence leads to a downregulation of IFN- $\kappa^{34,37}$

IFN $-\beta$ and IFN- $\gamma$ mRNA were found in the majority of cases in both epithelium and stroma compartments. Like the whole biopsy analysis, IFN- $\beta$ expression levels did not differ significantly between normal, dysplastic, and carcinoma groups in the epithelium (Jonckheere-Terpstra test for trend: $P=0.26$, one-sided for increase (n.s.) or decrease $P=0.73$, one-sided for decrease (n.s.)). However, a significant difference in the levels were found in the stroma between normal, dysplastic, and carcinoma cases (Jonckheere-Terpstra test for trend: $P=0.021$, one-sided). Similarly with IFN- $\gamma$, like the whole biopsy analysis, the expression levels did not differ significantly between normal, dysplastic, and carcinoma groups in the epithelium (Jonckheere-Terpstra test for trend: $P=0.19$, one-sided for increase (n.s.) or decrease $P=0.81$, one-sided for decrease (n.s)). However, a significant difference in levels was found in the stroma between normal, dysplastic, and carcinoma cases (Jonckheere-Terpstra test for trend: $P=0.041$, one-sided). Together, these experiments reveal significant increases in IFN $-\kappa,-\gamma$, and $-\beta$ mRNA levels in diseased compared with healthy cervical stroma. These findings correlated with an increased stromal infiltration of MCs (CD14) and DCs (CD11c) in diseased tissue as defined by immunofluorescence (Figure 3). The pronounced presence of these cells in diseased, compared with healthy tissue, suggests that these stromal populations are potentially 

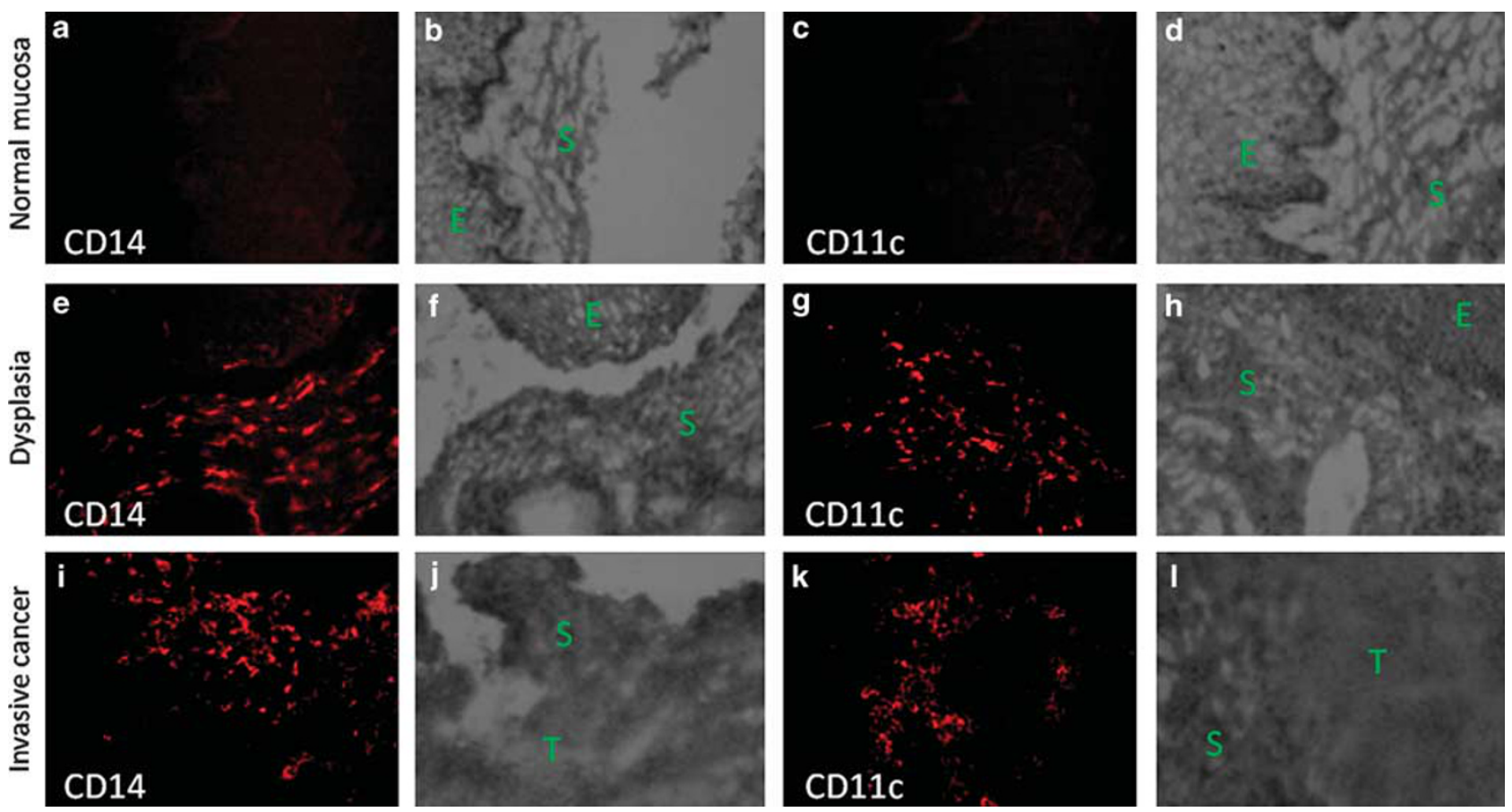

Figure 3 Infiltrating MCs and DCs in cervical tissue. Normal (a-d), dysplastic (e-h), and invasive cervical carcinoma (i-I) biopsy specimens were stained for infiltrating MCs (CD14) and DCs (CD11c). Altogether, 15 cases (normals, $n=5$; dysplasias, $n=7$; carcinomas, $n=3$ ) could be retrieved with sufficient remaining tissue. H\&E stains are shown in black and white. $\mathrm{E}$ denotes epithelium, $\mathrm{S}$ denotes stroma, and $\mathrm{T}$ denotes tumor. Representative cases of each diagnostic entity are shown. Original magnification was $\times 200$.

responsible for the increases in HPV-related IFN induction in cervical stroma.

\section{DISCUSSION}

In this study, using biopsy tissue and microdissected cervical epithelium and stroma, we analyzed the effect of HPV on IFN gene expression using qRT-PCR. To our knowledge, this study is the first of its kind to analyze IFN- $\kappa$ gene expression, in relation to other IFNs, in normal and HPV-infected pre-malignant and malignant ex vivo cervical tissue as well as using LCM to isolate cervical keratinocytes, the reservoir for HPV, from the underlying dermal stroma. Using normal, dysplastic, and cervical carcinoma whole cervical specimens, we have shown divergent trends among the type I IFNs analyzed, which highlight the unique and distinguishing properties of IFN $-\kappa$ in cervical tissue. Although trends exist with the analysis of whole cervical biopsy tissue, multiple cell types within the tissue sample may influence the analysis. Using microdissection to isolate cervical keratinocytes from stroma, which consists of a wide variety of cell types including DCs, fibroblasts and MCs, can provide insight into the cell-specific IFN response within the cervical microenvironment and this approach has been instrumental in obtaining the new and intriguing data described in the current paper.

IFN- $\kappa$ was detected more often in diseased tissue compared with normal whole biopsy tissue and, when detected,
IFN $-\kappa$ mRNA levels increased significantly with cervical disease progression, with highest levels observed in invasive carcinoma tissue. These results indicate that HPV has a role in its transcriptional activation. In contrast to IFN- $\kappa$, our whole biopsy data show that IFN- $\beta$ and IFN- $\gamma$ gene expression are found in all tissue samples but their expression levels do not differ statistically between groups.

Using LCM, IFN- $\kappa$ prevalence and gene expression levels differed between normal and diseased epithelium. IFN- $\kappa$ mRNA was absent in all dysplastic and carcinoma epithelial samples, indicating that HPV infection in cervical epithelium negatively influences IFN $-\kappa$ gene transcription. Although earlier results indicate that IFN- $\kappa$ is induced in epithelium in response to dsRNA, ${ }^{10}$ results of which we confirmed using NIKS and mucosal keratinocytes, DNA viruses have unique effects. Here, we show that IFN- $\kappa$ mRNA levels decrease in HPV16 + rafts during productive viral infection, further supporting the inhibitory role of HPV on IFN $-\kappa$ in cervical epithelium. Similarly, IFN- $\kappa$ mRNA levels have been found to be downregulated in primary human keratinocytes-expressing HPVE6 and E7, ${ }^{34,37}$ suggesting that both oncoproteins are involved in this process. In stark contrast, stromal expression of IFN- $\kappa$ was only present in diseased tissue, the expression of which accounts for the increase in IFN- $\kappa$ levels found in diseased whole biopsy tissue. Altogether, these data show that IFN- $\kappa$ is not being induced in the host cell infected by HPV or during a productive HPV infection, yet the 
presence of HPV indirectly triggers IFN- $\kappa$ expression in the cervical stroma. The presence of IFN- $\kappa$ exclusively in the stroma and not in the epithelium may be one factor, which impacts the persistence of HPV and ultimately the progression of the lesion. Discrepancies between the extent of IFN- $\kappa$ inhibition between ex vivo and in vitro material are not unexpected due to, for instance, differences in cell origin (keratinocytes or lymphoid populations) and tissue microenvironment (epithelium or stroma). With regard to IFN- $\beta$ and IFN- $\gamma$, mRNA levels in LCM-excised cervical epithelium did not decrease significantly as earlier literature suggests for HPV16 + keratinocytes. ${ }^{14,15,19}$ However, the seemingly constant levels may indicate the prohibition of a significant increase in the IFN response in the presence of HPV. In addition, discrepancies in IFN- $\beta$ and IFN- $\gamma$ results may be due to differences in sample integrity requirements between studies or explained by high intra-individual variability in expression levels. For IFN- $\gamma$, this variability seems not to be related to known single nucleotide polymorphisms within the IFN- $\gamma$ promoter as defined by sequence-specific primer PCRs (Zehbe et al, unpublished data).

Apart from elevated stromal IFN- $\kappa$ levels, LCM analysis also revealed increasing IFN- $\gamma$ and IFN- $\beta$ mRNA levels in diseased cervical stroma. Cytokine levels are generally tightly regulated signifying that the increases found in diseased cervical stroma likely beget significant consequences. The increased expression of IFN- $\gamma$ in diseased stroma may represent an intriguing mechanism of the HPV-related induction of IFN- $\kappa$. HPV in the epithelium could lead to an increased IFN- $\gamma$ production in infiltrating cells as part of the host-generated immune response. Rising IFN- $\gamma$ levels in infiltrate could trigger the observed transcriptional induction of IFN- $\beta$, as found in murine peritoneal macrophages, ${ }^{38}$ as well as IFN- $\kappa$, as it has been reported earlier that IFN- $\gamma$ can stimulate IFN- $\kappa$ expression in human ${ }^{39}$ and murine ${ }^{40}$ MCs. Coincidentally, levels of infiltrating DCs and MCs were more pronounced in diseased stromal tissue as defined by immunofluorescence in the current study. This corresponds well with earlier results, indicating that MCs and DCs and not fibroblasts and vessels, are IFN- $\kappa$ producers in the stroma. ${ }^{10}$ In addition, IFN- $\kappa$ mRNA was absent in raft cultures containing collagen and fibroblasts only (stroma equivalent), that is without keratinocytes, MCs or DCs in this study, which further supports the specific role of lymphoid populations in the stromal IFN $-\kappa$ induction. In line with IL-10 mRNA levels in this study, IL-10 has previously been found to be elevated in diseased cervical tissue ${ }^{41}$ and in peripheral blood mononuclear cells of patients with extensive cervical disease. ${ }^{42}$ However, the cause of the IL-10 elevation had not been addressed in these studies. Earlier results indicate that IFN $-\kappa$ strongly stimulates the production of IL-10 in MCs ${ }^{39}$ which could, at least in part, account for the concurrent changes in IL-10 in this study.

IFN- $\kappa$, although markedly upregulated in HPV + diseased cervical tissue, was not found in all diseased specimens analyzed. LCM analysis revealed the focal nature of IFN- $\kappa$ expression (data not shown) along with the importance of cervical stromal cells in IFN- $\kappa$ induction. The focal and low-expressing nature of IFN- $\kappa,{ }^{28}$ along with the potential differences in the occurrence of stromal cells among specimens, may explain why IFN- $\kappa$ was not detected in some HPV-positive cervical specimens. However, when IFN- $\kappa$ was detected, IFN- $\gamma$, IFN- $\beta$, and IL-10 were always present (data not shown).

HPV-associated cervical disease is a worldwide health concern. The recently approved prophylactic vaccine against common HR types of HPV is not a treatment for cervical or any other HPV-associated cancer and is not beneficial to individuals already infected with HPV or immunocompromised patients. As a consequence, there is a great need for studies, which characterize the effects of HPV on the immune system in efforts to improve on existing treatment options. Using LCM, our data show that IFN- $\kappa$ expression levels are not elevated in HPV-positive keratinocytes. Preliminary results reveal that IFN- $\kappa$ transfection into $\mathrm{SiHa}$ and HeLa HPV + cell lines, cell lines devoid of IFN $-\kappa$ mRNA, triggers cell apoptosis (Zehbe et al, unpublished data), suggestive of an IFN- $\kappa$-mediated anti-viral defense mechanism. Accordingly, taking into account the autocrine rather than paracrine signaling characteristics of IFN- $\kappa,{ }^{11}$ immunotherapeutic approaches involving focal administration of IFN- $\kappa$ could be an alternate treatment option for HPV-related diseases such as cervical, vulva, anal as well as head and neck cancers.

\section{ACKNOWLEDGEMENTS}

We thank Dr Margaret Anthes for help obtaining carcinoma tissue and the TBRHSC colposcopy team for help obtaining normal and dysplastic tissue samples and Dr Martin Mueller for the antibody against the HPV16-L2 protein. We also thank Christina Richard and Ashley Westerback for help with the completion of final experiments. This work was supported by the Northern Health Fund (to IZ), Grant \#: GA1-2005-009, a Discovery Grant (to IZ) from the Natural Sciences and Engineering Research Council of Canada (NSERC), Grant \#: 355858-2008 and by an Ontario Graduate Scholarship (to $C D)$.

\section{DISCLOSURE/CONFLICT OF INTEREST}

The authors declare no conflict of interest.

1. Polman $\mathrm{CH}, \mathrm{O}^{\prime}$ Conner PW, Havrdova $\mathrm{E}$, et al. A randomized, placebocontrolled trial of natalizumab for relapsing multiple sclerosis. N Engl J Med 2006;354:899-933.

2. De Maeyer E, De Maeyer-Guignard J. Interferons. In: Thompson A (ed.) The Cytokine Handbook, 3rd edn., Academic Press: San Diego, CA, USA, pp 491-516.

3. Gerein V, Rastorguev E, Gerein J, et al. Use of interferon-alpa in recurrent repiratory papillomatosis: 20 year follow-up. Ann Otol Rhinol Laryngol 2005;114:463-471.

4. Scheinfeld N, Lehman DS. An evidence-based review of medical and surgical treatment of genital warts. Dermatol Outline J 2006;12:5.

5. Isaacs A, Lindenman J. Virus interference I. The interferon. Proc R Soc 1957;147:258-267.

6. Takaoka A, Hayakawa $S$, Yani $H$, et al. Integration of interferon- $\alpha / \beta$ signalling to p53 responses in tumor suppression and antiviral defence. Nature 2003;424:516-523. 
7. Stetson DB, Medzhitov R. Type I interferons and host defense. Immunity 2006;25:373-381.

8. Pestka S, Langer JA, Zoon KC, et al. Interferons and their actions. Annu Rev Biochem 1987;56:727-777.

9. Bach EA, Aguet $\mathrm{M}$, Schreiber RD, et al. The IFN receptor: a paradigm for cytokine receptor signaling. Annu Rev Immunol 1997;15:563-591.

10. Lafleur DW, Nardelli B, Tsareva T, et al. Interferon- $\kappa$, a novel type I interferon expressed in human keratinocytes. J Biol Chem 2001;276:39765-39771.

11. Buontempo PJ, Jubin RG, Buontempo CA, et al. Antiviral activity of transiently expressed IFN- $\kappa$ is cell-associated. J Inter Cyto Res 2006:52:40-52.

12. Manns MP, McHutchison JG, Gordon SC, et al. Albrecht Interferon alfa-2b plus ribavirin compared with interferon alfa2b plus ribavirin for initial treatment of chronic hepatitis $\mathrm{C}$ : a randomised trial. Lancet 2001;358:958-965.

13. Scarponi C, Nardelli B, LaFleur DW, et al. Analysis of IFN- $\kappa$ expression in pathological skin conditions: downregulation in psoriasis and atopic dermatitis. J Inter Cyto Res 2006;26:133-140.

14. Park JS, Kim EJ, Kwon HJ, et al. Inactivation of interferon regulator factor-1 tumor suppressor protein by HPV E7 oncoprotein. J Biol Chem 2000;275:6764-6769.

15. Ronco LV, Karpova AY, Vidal M, et al. Human papillomavirus 16 E6 oncoprotein binds to interferon regulatory factor- 3 and inhibits its transcriptional activity. Genes Dev 1998;12:2061-2072.

16. Barnard P, McMillan NA. The human papillomavirus E7 oncoprotein abrogates signalling mediated by interferon-alpha. Virology 1999;259:305-313.

17. Li S, Labrecque S, Gauzzi MC, et al. The human papilloma vrus (HPV)-18 E6 oncoprotein physically associates with Tyk2 and impairs Jak-STAT activation by interferon- $\alpha$. Oncogene 1999;18:5727-5737.

18. Chang YE, Laimins LA. Microarray analysis identifies interferoninducible genes and stat- 1 as major transcriptional targets of human papillomavrus type 31. J Virol 2000;74:4174-4182.

19. Nees M, Geoghegan JM, Hyman T, et al. Papillomavirus type 16 oncogenes downregulate expression of interferon-responsive genes and upregulate proliferation-associated and NF-kB-responsive genes in cervical keratinocytes. J Virol 2001;75:4283-4296.

20. Bachmann A, Hanke B, Zawatzky R, et al. Disturbance of tumor necrosis factor alpha-mediated beta interferon signalling in cervical carcinoma cells. J Virol 2002;76:280-291.

21. de Gruijl TD, Bontkes HJ, van den Muysenberg AJ, et al. Differences in cytokine mRNA profiles between premalignant and malignant lesions of the uterine cervix. Eur J Cancer 1999;35:490-497.

22. Gey A, Kumari P, Sambandam A, et al. Identification and characterisation of a group of cervical carcinoma patients with profound downregulation of intratumoral type I (IFN- $\gamma$ ) and type 2 (IL-4) cytokine mRNA expression. Eur J Cancer 2003;39:595-603.

23. Gravitt PE, Peyton $C L$, Alessi $T Q$, et al. Improved amplification of genital human papillomaviruses. J Clin Microbiol 2000;38:357-361.

24. Goleski VA, Dawood M, Smart G, , et al., The Canadian Women's HIV Study Group. A microsphere based multiplexed assay for genotyping 46 mucosal human papillomavirus types. Proceedings from the Canadian Association for Clinical Microbiology and Infectious Diseases Annual Conference, 14-18 March 2007; Halifax Canada.

25. Goleski VA, Dawood M, Ratnam S, et al. Luminex-based assay for multiplex genotyping of 45 mucosal human papillomavirus types.
Proceedings from the European Research Organisation on Genital Infection and Neoplasia Conference, 12-15 November 2008; Nice France.

26. Zehbe I, Rylander E, Edlund K, et al. Detection of human papillomavirus in cervical intra-epithelial neoplasia, using in situ hybridization and various polymerase chain reaction techniques. Virchows Arch 1996;428:151-157.

27. Culling CFA, Allison RT, Barr WT. Cellular pathology techniques. In: Culling CFA, Allison RT, Barr WT (eds). Microorganisms, 4th edn. Butterworths: London, PA, 1985, pp 55-59.

28. Allen-Hoffmann BL, Schlosser SJ, Ivarie CA, et al. Normal growth and differentiation in a spontaneously immortalized near-diploid human keratinocyte cell line, NIKS. J Invest Dermatol 2000;114: 444-455.

29. Zehbe I, Richard C, DeCarlo CA, et al. Human papillomavirus 16 E6 variants in their dysregulation of human keratinocyte differentiation and apoptosis. Virol 2009;383:69-77.

30. Flores ER, Allen-Hoffmann BL, Lee $\mathrm{D}$, et al. The human papillomavirus type $16 \mathrm{E} 7$ oncogene is required for the productive stage of the viral life cycle. J Virol 2000;74:6622-6631.

31. DeCarlo CA, Escott NG, Werner J, et al. Gene expression analysis of interferon kappa in laser capture microdissected cervical epithelium. Anal Biochem 2008;381:59-66.

32. Fleiss JL, Levin B, Cho Paik M. Statistical Methods for Rates and Proportions. John Wiley \& Sons: New Jersey, USA, 2003.

33. Hollander M, Wolfe DA. Nonparametric Statistical Methods. John Wiley \& Sons: New Jersey, USA, 1999.

34. Dell'Oste V, Azzimonti B, Mondini M, et al. Altered expression of UVBinduced cytokines in human papillomavirus-immortalized epithelial cells. J Gen Virol 2008;89:2461-2466.

35. Woodworth CD. HPV innate immunity. Front Biosci 2002;7: d2058-d2071.

36. Richard C, Lanner C, Naryzhny SN, et al. The immortalizing and transforming ability of two common human papillomavirus 16 E6 variants with different prevalences in cervical cancer. Oncogene 2010, doi:10.1038/onc.2010.93 (in press).

37. Rincon-Orozco B, Halec G, Rosenberger S, et al. Epigenetic silencing of interferon- $\kappa$ in human papillomavirus type 16-positive cells. Cancer Res 2009;69:8718-8725.

38. Gessani S, Belardelli F, Pecorelli A, et al. Bacterial lipopolysaccharide and gamma interferon induce transcription of beta interferon mRNA and interferon secretion in murine macrophages. J Virol 1989;63: 2785-2789.

39. Nardelli B, Zaritskaya L, Semenuk M, et al. Regulatory effect of IFN- $\kappa$, a novel type I IFN, on cytokine production by cells of the innate immune system. J Immunol 2002;169:4822-4830.

40. Vassileva G, Chen SC, Zeng M, et al. Expression of a novel murine type I IFN in the pancreatic islets induces diabetes in mice. J Immunol 2003;170:5748-5755.

41. El-Sherif AM, Seth R, Tighe PJ, et al. Quantitative analysis of IL-10 and IFN-gamma mRNA levels in normal cervix and human papillomavirus type16 associated cervical precancer. J Path 2001;195:179-185.

42. Clerici $M$, Merola $M$, Ferrario $E$, et al. Cytokine production patterns in cervical intraepithelial neoplasia: association with human papillomavirus infection. J Nat Cancer Inst 1997; 89:245-250. 